Maria del C. Valdés Hernández

Karen J. Ferguson

Francesca M. Chappell

Joanna M. Wardlaw

\section{New multispectral MRI data fusion technique for white matter lesion segmentation: method and comparison with thresholding in FLAIR images}

Received: 6 August 2009

Accepted: 6 December 2009

Published online: 16 February 2010

(C) The Author(s) 2010.

This article is published with open access at Springerlink.com
Electronic supplementary material The online version of this article (doi:10.1007/ s00330-010-1718-6) contains supplementary material, which is available to authorized users.

M. d. C. Valdés Hernández •

K. J. Ferguson - F. M. Chappell .

J. M. Wardlaw

SFC Brain Imaging Research Centre,

SINAPSE Collaboration,

Division of Clinical Neurosciences,

School of Molecular and Clinical

Medicine, University of Edinburgh,

Edinburgh, UK

M. d. C. Valdés Hernández •

J. M. Wardlaw

Centre for Cognitive Ageing

and Cognitive Epidemiology,

University of Edinburgh,

Edinburgh, UK

\author{
M. d. C. Valdés Hernández $(\square)$ \\ SFC Brain Imaging Research Centre, \\ Image Analysis Lab, Division of \\ Clinical Neurosciences, Western \\ General Hospital, University of \\ Edinburgh, \\ Crewe Road, \\ Edinburgh, EH4 2XU, UK \\ e-mail: mvhernan@staffmail.ed.ac.uk \\ Tel.: +44-131-5373669 \\ Fax: +44-131-3325150
}

Abstract Objective: Brain tissue
segmentation by conventional
threshold-based techniques may have
limited accuracy and repeatability in
older subjects. We present a new
multispectral magnetic resonance
(MR) image analysis approach for
segmenting normal and abnormal
brain tissue, including white matter
lesions (WMLs). Methods: We
modulated two $1.5 \mathrm{~T}$ MR sequences in
the red/green colour space and calcu-
lated the tissue volumes using mini-
mum variance quantisation. We tested
it on 14 subjects, mean age $73.3 \pm$
10 years, representing the full range
of WMLs and atrophy. We compared

the results of WML segmentation with those using FLAIR-derived thresholds, examined the effect of sampling location, WML amount and field inhomogeneities, and tested observer reliability and accuracy. Results: FLAIR-derived thresholds were significantly affected by the location used to derive the threshold $(P=0.0004)$ and by WML volume $(P=0.0003)$, and had higher intrarater variability than the multispectral technique (mean difference \pm SD: $759 \pm 733$ versus $69 \pm 326$ voxels respectively). The multispectral technique misclassified 16 times fewer WMLs. Conclusion: Initial testing suggests that the multispectral technique is highly reproducible and accurate with the potential to be applied to routinely collected clinical MRI data.

Keywords White matter lesions · Leukoaraiosis · Ageing · Tissue segmentation - MRI · Multispectral segmentation . Threshold segmentation - FLAIR

\section{Introduction}

White matter lesions (WMLs) are commonly observed on MR images of older people. Their association with declining cognitive ability, future stroke risk and dementia is well recognised. WMLs can be assessed using subjective rating scales, but the wider availability of more detailed MR brain imaging has led to increasing attempts to measure WML volumes.
Many studies measure WML volume using a single MR sequence [1-6]. Some delineate WMLs using a regiongrowing algorithm based on pixel intensity in FLAIR images, followed either by manual editing $[1,6,7]$ or by mixture modelling-based algorithms [4]. Other methods use local thresholds in each slice to mark the lesions and borders [5]. While these techniques are typically methodologically simple, the resulting segmentations are prone to operator bias, have low precision and are time consuming [8]. 
Combining sequences may increase the reliability of tissue segmentation and reduce the reliance on observer input. Such approaches include probabilistic methods [9], neural networks [10], genetic algorithms [11], fuzzy segmentation [12], Parzen windows [13], the Markov field random model, and the expectation-maximisation algorithm [14]. These methods demand complex computational efforts to achieve good reproducibility and publications often fail to mention practicalities such as processing time or the amount of manual editing required. Finally, it is unclear if atrophy and focal lesions, which are common in older subjects and may interfere with the image processing algorithms, affect the accuracy of these techniques.

We developed a completely new approach to overcome these difficulties for a study of ageing [15]. We fused pairs of different MR sequences and modulated them in the redgreen (RG) colour space thereby enhancing tissue discrimination. We compared this with WML segmentation performed by local thresholding of a FLAIR sequence, a commonly used approach.

\section{Materials and methods}

Subjects

We selected MR data sets from two previous studies: one of normal male volunteers aged 65-70 years and one of patients with mild stroke aged $50-90$ years $[16,17]$. The MR images were rated for WMLs using validated qualitative rating scales [18] by two experienced raters (JMW, KJF), blinded to each other [19]. Using these ratings, we randomly selected 14 subjects that represented the full range of WMLs and brain atrophy: eight from the normal male volunteers aged 65-70 years [20], and six from the patients with mild non-disabling stroke aged 50-90. [17] The average age of the 14 subjects was $73.3 \pm 10$ years. Both studies had Ethics Committee approval, and all subjects gave informed consent.

\section{MRI}

All MR images were obtained on a 1.5T GE Echospeed MRI system. These included sagittal T1-weighted (T1W), axial T2-weighted $(\mathrm{T} 2 \mathrm{~W}), \mathrm{T} 2 *$-weighted $(\mathrm{T} 2 * \mathrm{~W})$ and FLAIR images (sequence details given in Table 1). The DICOM files were converted to data ANALYSE format. [21] The images from the stroke study were linearly interpolated from matrix $256 \times 192$ to $256 \times 256$.

\section{Definition of WMLs}

We identified WMLs as diffuse patchy areas in the white matter (WM), basal ganglia or brain stem, larger than $3 \mathrm{~mm}$ in diameter and hyperintense with respect to normal white and grey matter on T2W and FLAIR images [18].

\section{Threshold-based WML segmentation on FLAIR}

FLAIR-based thresholding relies on the change in tissue intensity at the WML boundary. To determine the best and most subject-specific thresholds, we sampled signal intensity in normal-appearing white matter using Analyze 8.1 software [21]. We placed two regions of interest (ROIs), each $5 \times 5 \mathrm{~mm}$ square, bilaterally, in normal-appearing frontal white matter on all slices on which frontal white matter was visible, from the inferior frontal lobes to near

Table 1 MR sequence details for the two studies that contributed image data to the present analyses

\begin{tabular}{llll}
\hline Study & Ageing brain & Mild stroke (protocol 1) & Mild stroke (protocol 2) \\
\hline TR/TE (ms) T1W & $440 / 9$ & $440 / 9$ & $440 / 9$ \\
TR/TE (ms) T2W & $6,300 / 106.34$ & $6,300 / 106.13$ & $6,300 / 107.64$ \\
TR/TE (ms) T2*W & $620 / 15$ & $2000 / 9.912$ & $620 / 15$ \\
TR/TE/TI (ms) FLAIR & $9,002 / 149.5 / 2,200$ & $10,002 / 147 / 2,500$ & $9,002 / 147 / 2,200$ \\
Pixel bandwidth T1W & 122.11 & 125 & 125 \\
Pixel bandwidth T2W & 61.035 & 61.035 & 61.035 \\
Pixel bandwidth T2*W & 97.656 & 81.38 & 97.656 \\
Pixel bandwidth FLAIR & 122.07 & 122.07 & 122.07 \\
Matrix T1W, T2*W, FLAIR & $256 \times 256$ & $256 \times 256$ & $256 \times 192$ \\
No. slices T1W & 33 & 20 & 20 \\
No. slices T2W, T2*W, FLAIR & 24 & 20 & 20 \\
Slice thickness (mm) T1W & 5 & 5 & 5 \\
Slice thickness (mm) T2W, T2*W, FLAIR & 5.6 & 5 & 5 \\
Inter-slice gap (mm) T1W & 5 & 6.5 & 6.5 \\
Inter-slice gap (mm) T2W, T2*W, FLAIR & 5.6 & 6.5 & 6.5 \\
Spatial resolution T1W/T2W, T2*W, FLAIR & $1.0714 / 1.7188$ & $1.0714 / 0.9375$ & $1.0714 / 1.0714$ \\
\hline
\end{tabular}


the vertex. We grouped the slices into 12 adjacent pairs and calculated the mean ROI intensity for each slice pair. The subject's WML threshold was set as six standard deviations (SDs) above the mean intensity, a method previously tested in our lab. This produced 12 thresholds per subject, from inferior frontal to near vertex.

We produced three WML masks per patient using the maximum, minimum and mean of these 12 thresholds. To identify WMLs, we placed an auto-trace seed in normal white matter on a reference image and highlighted automatically all areas of intensity higher than the calculated threshold [3]. The WML edges were then automatically delineated by a region-growing algorithm from seeds placed manually on the lesions and set at the intensity range: $\min =$ calculated threshold, $\max =$ maximum intensity. We performed minimal manual editing to remove erroneously included areas (e.g. normal caudate nucleus) and repeated the above process without excluding any erroneous areas to test the influence of observer intervention. We also examined whether the FLAIR-derived thresholds were influenced by slice position by comparing the threshold values of the 12 slice pairs.

Multispectral data fusion and minimum variance quantisation (MVQ) technique

To develop our new technique, we selected two MR sequences that produced different intensity ranges in the tissues to be segmented and optimised their grey scale intensity range using Analyze 8.1 [21]. By trial and error, we found that the coloured $\mathrm{RG}$ combination of $\mathrm{T} 2 * \mathrm{~W}$ and FLAIR was good for differentiating WMLs and CSF and the combination of T1 W and T2W was good for recognising
CSF and white matter (Fig. 1). We registered the images using the 3-D Surface Registration module in Analyze 8.1, a surface-matching algorithm that is fast and robust, even in the presence of image noise [21]. We used T2W images as 'base' objects and 'matched' all other images (T1W, T2*W, FLAIR) to it. We transformed the sequence pairs to the redgreen $(\mathrm{RG})$ colour space [22], putting $\mathrm{T} 2 * \mathrm{~W}$ through the red channel and FLAIR through the green channel so that the resulting coloured volume contained the information of the fused T2*W and FLAIR volumes. We performed a similar process for the T1W and T2W sequence pair. We used the $\mathrm{RG}$ colour space because the human eye cones are most sensitive to the frequencies in this range of the visible spectrum [23]. Wyszecki and Stiles [24] measured luminous efficiency using six different techniques and demonstrated that the maximum luminous efficiency of the human eye is reached at wavelengths of around $550 \mathrm{~nm}$, remaining at $80 \%$ or above up to the maximum value in the interval from $525 \mathrm{~nm}$ (green) to $670 \mathrm{~nm}$ (dark red).

To extract the brain from the fused RG volumes for further processing, we used $\mathrm{T} 2 * \mathrm{~W}$ images, as this provides good differentiation between brain-CSF and inner skull table and extracted the brain using the Object Extraction Tool in Analyze 8.1 [21]. To segment and quantify the WML and tissue volumes from the RG-modulated image, we developed a software module using MATLAB (Fig. 2) [25]. The initial fused volume had 65,535 different levels in the RG space, so a minimum variance quantisation (MVQ) algorithm, which uses Floyd-Steinberg's 'error diffusion dither principle' [26] implemented by the MATLAB function 'rgb2ind' [25] was used to reduce colour depth to a more manageable number of levels. Orchard and Bouman showed MVQ to be the optimal clustering method because more colour map entries are allocated to densely
Fig. 1 Central axial slice of a subject displaying the registration and fusion processes for MCMxxxVI. First row, left to right: $\mathrm{T} 2 \mathrm{~W}, \mathrm{~T} 1 \mathrm{~W}$ images and their fusion and modulation in red and green channels respectively; second row, left to right: $\mathrm{T} 2 * \mathrm{~W}$ and FLAIR images and their fusion and modulation in red and green channels

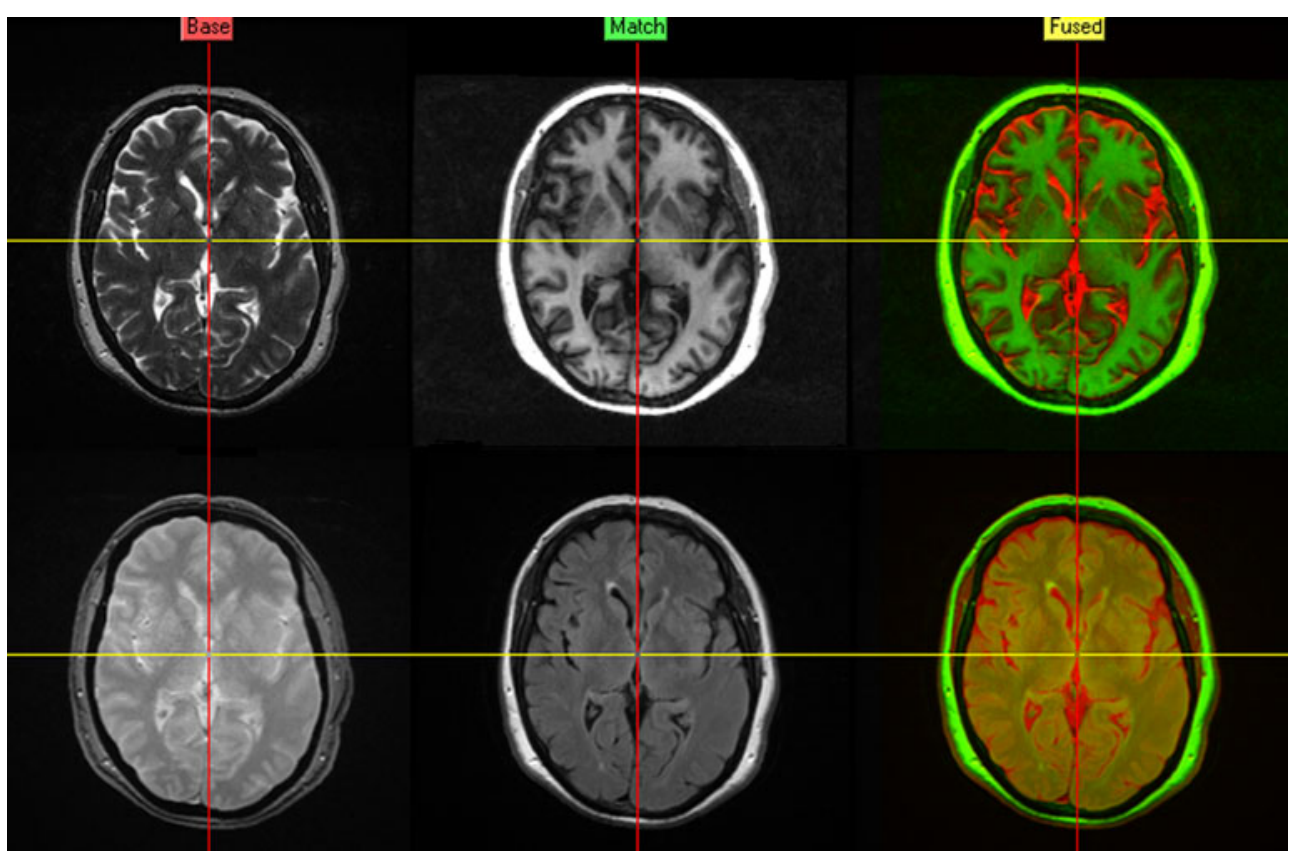




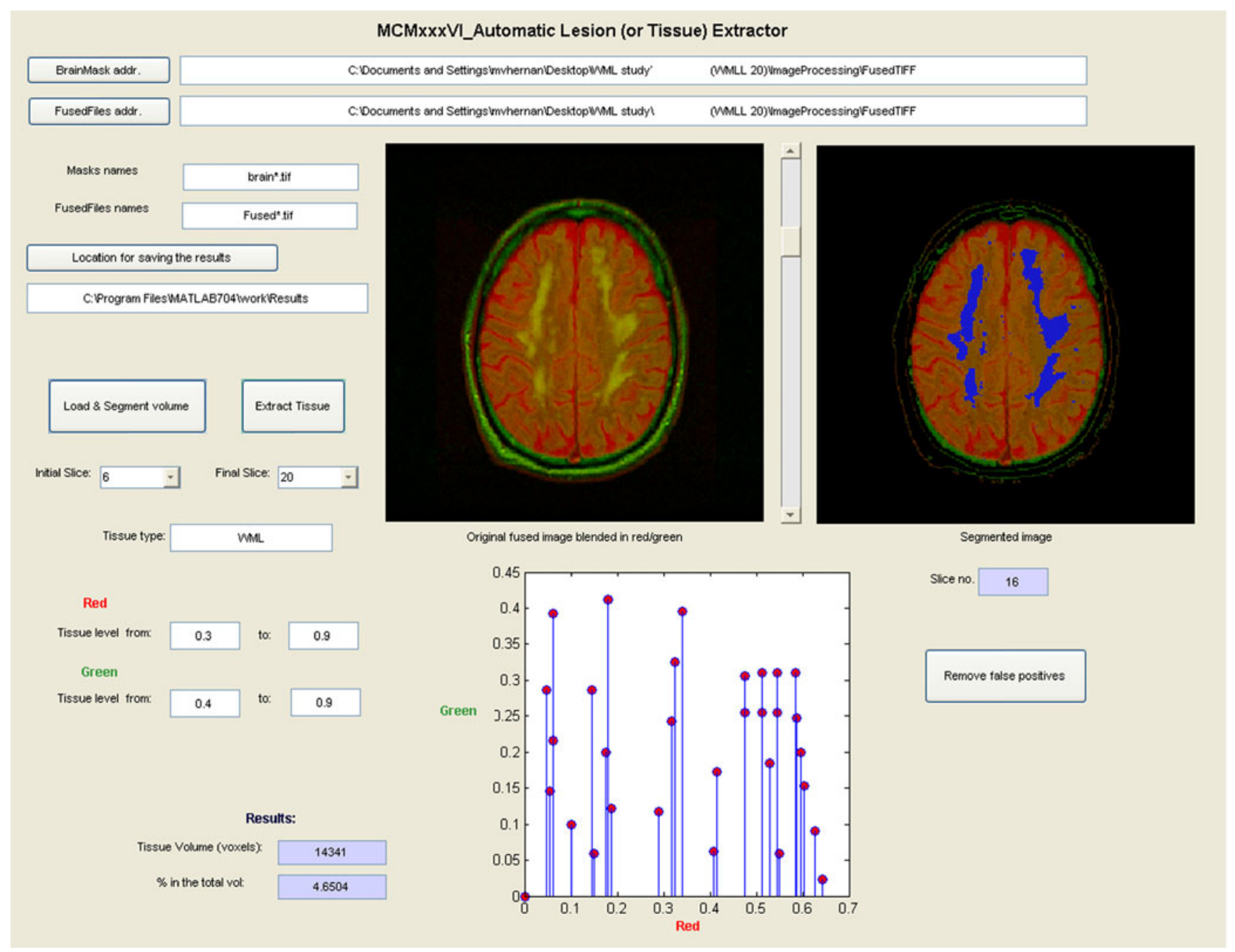

Fig. 2 Screen capture of the software module that performs the segmentation, showing a slice of a fused volume, the result of segmenting the WMLs in the slice, and the clusters obtained from the MVQ algorithm mapped in the normalised RG space

populated areas in the colour space, and fewer entries are allocated to infrequent colours [27]. They demonstrated that MVQ achieved a time complexity of $N \log _{2} M$, where $N$ is the number of pixels in the image and $M$ the number of the desired clusters in which the coloured image is dithered. Experimentally we determined that reducing the 65,535 levels to 32 clusters provided good reproducibility of all tissue classes (see graph, Fig. 2).

We then selected the clusters corresponding to the tissue of interest, e.g. WMLs. Moving the cursor over the WMLs in the quantised images showed the corresponding RG coordinate values on the graph (Fig. 2). One, or at most a few, prototypical clusters per subject are sufficient to identify the maximum and minimum RG coordinates of the required tissue on each axis. The software then automatically constructs the tissue segmentation mask from the quantised volume. Minimal manual editing was required to remove minimal false positive WMLs. In this paper we compare the WML volumes before and after manual editing. The video (multimedia file, see the ESM) demonstrates the process of extracting CSF and WMLs.

We named this method "MCMxxxVI" which stands for multispectral colouring modulation and variance identification and also represents the number 1936, reflecting the Lothian Birth Cohort 1936 study for which it was developed.

\section{Observer variation and statistical analysis}

Two trained observers (MVH and KJF), independently and blinded to each other's results, performed WML segmentation using the MCMxxxVI method and FLAIR-derived thresholds (comparisons summarised in Table 2). The FLAIR thresholds derived from the three most caudal slice 
Table 2 Comparisons performed to evaluate the segmentation methods

\begin{tabular}{lccc}
\hline Segmentation method & Intra-observer reliability & Inter-observer reliability & Visual assessment \\
\hline MCMxxxVI manually removing false positive WMLs & $\checkmark$ & $\checkmark$ & $\checkmark$ \\
MCMxxxVI without removing false positive WMLs & - & - & $\checkmark$ \\
Thresholding in FLAIR manually removing false positive WMLs & $\checkmark$ & $\checkmark$ & $\checkmark$ \\
Thresholding in FLAIR without removing false positive WMLs & - & - & $\checkmark$ \\
\hline
\end{tabular}

pairs were discarded from further analyses as they were clearly different (Fig. 3). We calculated the minimum (threshold 1) and maximum (threshold 2) per patient and also a single threshold equal to the average of all 168 thresholds (9 slice pairs, 14 subjects). The single threshold was evaluated because some studies use only one threshold for segmenting WMLs in all patients [2]. The resulting three WML masks were visually assessed, blind to threshold value and to each other, for accuracy by an experienced neuroradiologist (JMW) who determined the number of false positive and partial and total false negative WMLs per brain region.

We expressed the WML volumes derived from each method in voxels and calculated the mean, maximum and minimum differences using Bland-Altman analyses [28]. The effect of slice location and WML load on FLAIRderived thresholds was explored using a linear mixed model and expressed as means with 95\% confidence intervals (CI). Separate simple linear regression analyses were used to test for the effect of observer variation of $\mathrm{RG}$ boundary values on WML volume. The results of the intraand inter-observer reliability tests are expressed in voxels, not cubic millimetres because of the inter-slice gap $(5 \mathrm{~mm})$, i.e. we did not at this stage apply stereology to interpolate between slices. Finally, we evaluated the effect of motion artefact and magnetic field heterogeneities [29] on WML volume measurement $[13,30]$.

\section{Results}

FLAIR-derived WML thresholds were influenced by both slice position and WML volume. The FLAIR derived threshold decreased by $2.52(3.90,1.14)$ units between sequential adjacent slice pairs from brain stem to vertex $(P=$ 0.0004 , Fig. 3), and increased by 3.14 (95\% CI 1.79, 4.48) units for every voxel increase in WML volume $(P=0.0003$, Table 3 ). In comparison, WML volume had no effect on RG levels or on WML volume using the MCMxxxVI (Table 3). FLAIR-derived thresholds were also affected by image contrast levels, indeed the image of one subject differed very much from the others because the contrast was extremely low (data not shown). Image contrast had no effect on MCMxxxVI-derived volumes.

There was good intra-rater repeatability for WML volumes calculated using the same FLAIR-derived threshold each time (SD of \pm 734 voxels) and with MCMxxxVI (SD of \pm 326 voxels) but not when an individual new FLAIR-derived threshold was used each time to segment the WMLs (SD of $\pm 9,924$ voxels, Table 4). This lowest intrarater variation for the MCMxxxVI method occurred even without manual correction to remove any false positives/ negatives (Table 4). We did not measure the inter-rater variability of the FLAIR-derived threshold techniques, as its intra-rater measurement variability was so much higher than the inter-rater variability of the MCMxxxVI method.
Fig. 3 Threshold (Th) values calculated from each pair of slices for each subject. Threshold 1 is from the brain slice nearest the skull base and threshold 12 from the slice nearest the vertex

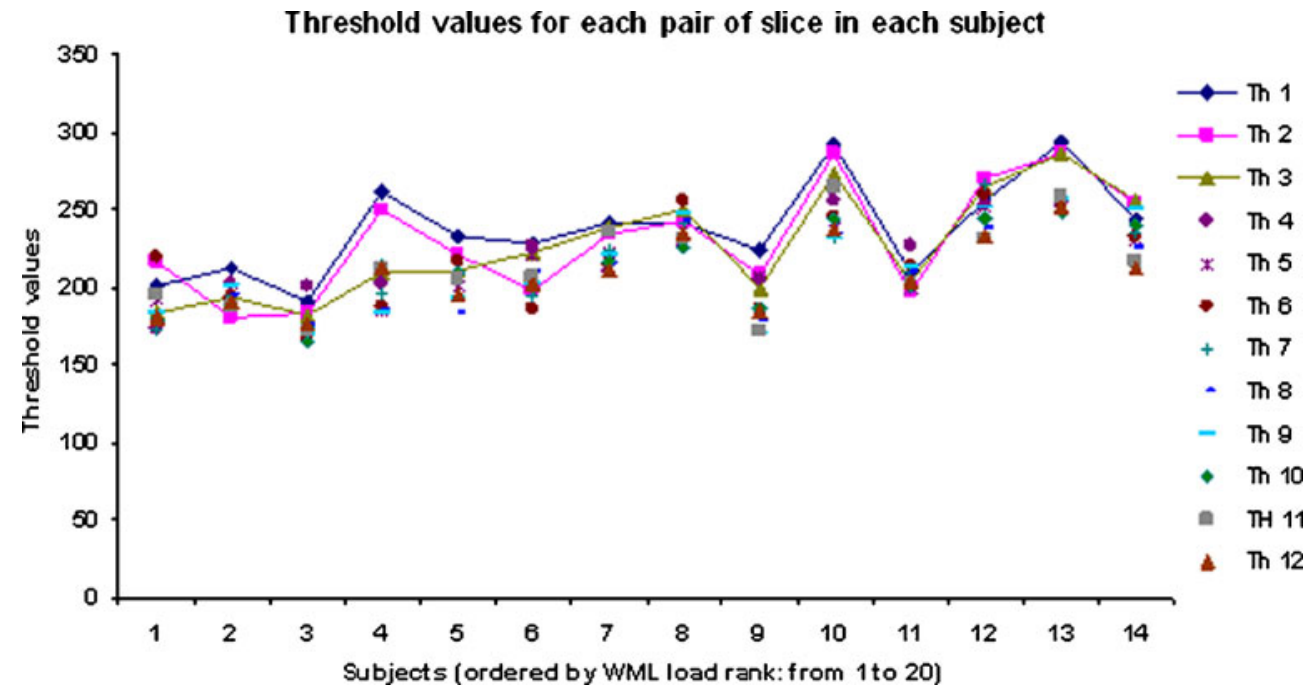


Table 3 The effect of WML volume on FLAIR-derived thresholds (tested with linear regression analyses) and on the red/green levels for the MCMxxxVI method (tested with univariate linear regression analyses)

\begin{tabular}{llll}
\hline Parameter & Effect per unit in WML load & $95 \%$ Confidence interval & $P$ value \\
\hline Mean threshold per subject & 3.36 & $(1.63,5.09)$ & 0.0012 \\
R min level (analyst 1) & 0.0023 & $(-0.0022,0.0069)$ & 0.27 \\
R min level (analyst 2) & -0.00030 & $(-0.0063,0.0057)$ & 0.91 \\
G min level (analyst 1) & 0.0014 & $(-0.0019,0.0047)$ & 0.36 \\
G min level (analyst 2) & -0.00011 & $(-0.0061,0.0059)$ & 0.97 \\
\hline
\end{tabular}

Visual comparison of WMLs identified using FLAIRderived thresholds and MCMxxxVI showed that the range (y axis) of errors was 16 times greater for FLAIR-derived thresholds than for MCMxxxVI, i.e. both false positives and negatives were very frequent with the FLAIR-derived thresholds and increased with increasing WMLs but were rare with MCMxxxVI (Fig. 4). WML segmentation errors differed with brain region in both methods (data not shown), but the MCMxxxVI approach misclassified few lesions except in the pons, where a few errors occurred due to CSF flow artefacts.

\section{Discussion}

We present a novel multispectral image processing method, MCMxxxVI, for segmenting normal brain tissue and WMLs. We have demonstrated that slice position in the brain, WML load and focal lesions affect the accuracy of WML volume determined using FLAIR-derived thresholds but have little influence on the MCMxxxVI method. In this test sample of 14 subjects, deliberately chosen to represent a wide range of WMLs and accompanying features of ageing such as atrophy, the MCMxxxVI had the best intrarater reliability. In contrast to FLAIR-derived thresholds, MCMxxxVI was not affected by focal signal extremes, number of WMLs, bias field heterogeneities or movement artefacts. It was fast and easy to use. Further testing is now warranted in much large populations.

The MCMxxxVI method uses an area of the two dimensional $R G$ space that combines information from two different MR sequences chosen to optimise differences in signal between tissue types, i.e. it enhances their radiological properties. In addition to segmenting WMLs, the multispectral technique also enables extraction of intracranial volume, whole brain, CSF, grey matter and normal white matter volumes using different combinations of MR sequences. We used T2* and FLAIR to segment CSF and WMLs and T2 and T1 to segment normal grey and white matter. An added advantage is that use of two or more images in combination reduces the effects of distortions and noise found in a single sequence. Thus a FLAIR image with movement artefact or bias field heterogeneity impairs measurement of WML volume using a threshold, potentially rendering that subject's data unusable. However, as it is unlikely that all sequences would be badly affected by movement or bias field heterogeneity, combining the FLAIR with a less movement-affected or bias fieldaffected $\mathrm{T}_{2} *$ in the MCMxxxVI method attenuates any adverse effects present in one sequence and means that the subject's data can still be used.

The strengths of our study are that we have compared our method with a conventional WML volume measurement method and examined the sources of error and variation with computational as well as visual assessment. We used carefully chosen images representing a wide range of WMLs and other abnormalities from normal older subjects and patients with stroke. To our knowledge, no previous studies have assessed WML segmentation as comprehensively. The weaknesses include the small sample, the use of data from one scanner (albeit with different sequence details) and the prototypical stage of the MCMxxxVI method placing it at a disadvantage compared with more established methods such as FLAIR thresholding. We have

Table 4 Intra- and inter-observer reliability of segmenting WMLs using MCMxxxVI and FLAIR-derived thresholds

\begin{tabular}{llllll}
\hline Measurements (voxels) & \multicolumn{2}{l}{ For the same analyst } & & \multicolumn{2}{l}{ For two analysts } \\
\cline { 2 - 4 } & Mean \pm SD & Min. & Max. & Mean \pm SD & Min. Max. \\
\hline $\begin{array}{l}\text { FLAIR-derived threshold: differences between two WML volume } \\
\text { measurements using the same threshold }\end{array}$ & $759.00 \pm 733.53$ & 17 & 2,628 & - \\
$\begin{array}{l}\text { FLAIR-derived threshold: differences between two WML volume } \\
\text { measurements using a different threshold for each measurement }\end{array}$ & $310.00 \pm 9,923.98$ & 3,664 & 16,444 & - \\
MCMxxxVI: differences between two WML volume measurements & $69.00 \pm 326.08$ & 1 & 698 & $761.00 \pm 822.21$ & - \\
\hline
\end{tabular}

Same threshold indicates that the same threshold was applied to the subject each time. Different threshold indicates that an individual threshold was derived for that subject each time the WML volume was measured (which is a closer reflection of the usual practice) 
Fig. 4 Number of errors in WML segmentation identified by an experienced neuroradiologist when a a single threshold was applied to all FLAIR images and $\mathbf{b}$ the multispectral MCMxxxVI method was applied to the sample (horizontal axis) ordered by WML load. Note the difference in $y$-axis values
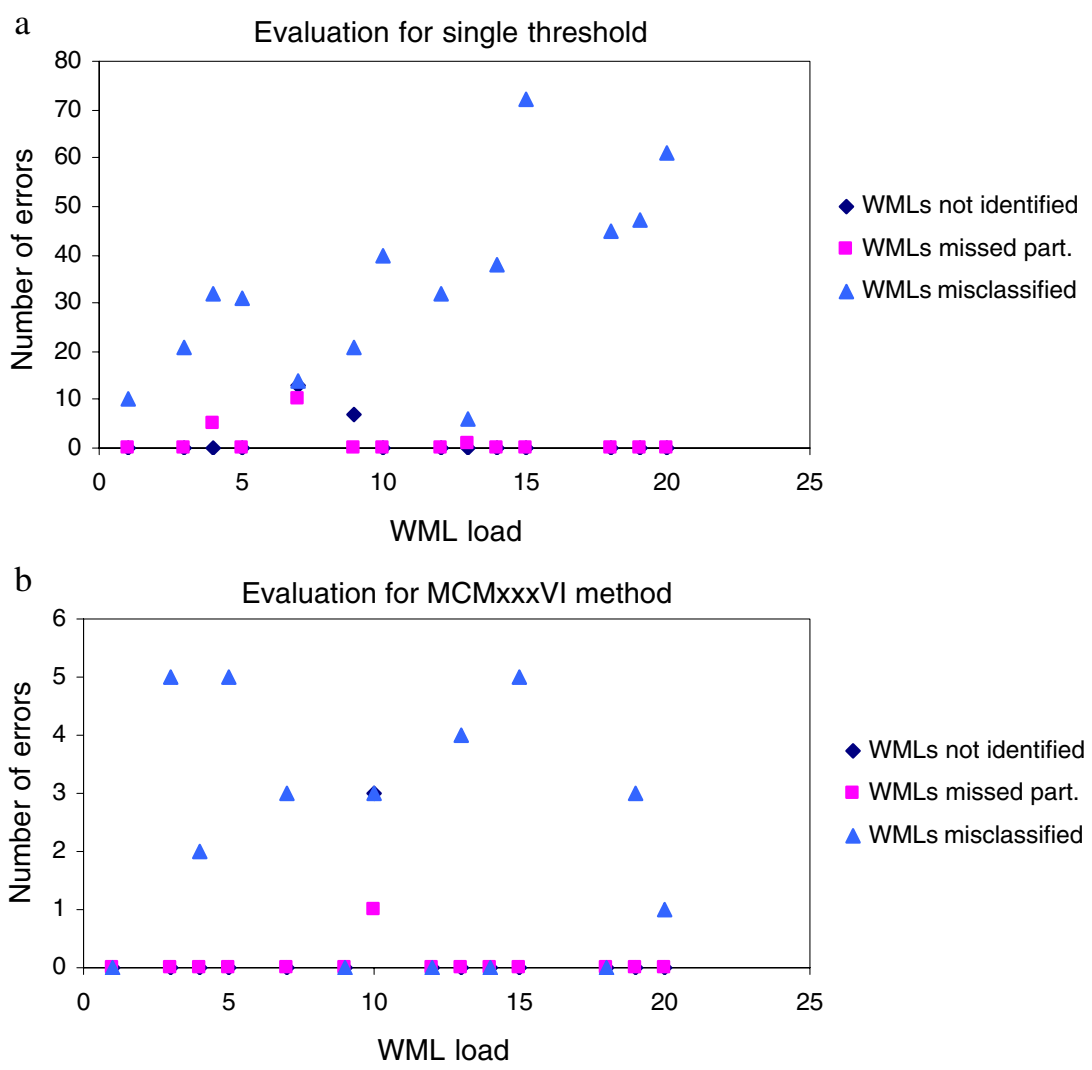

not performed extensive testing of the influence of WML volume on RG space boundaries or methods to remove the minor misclassification of WMLs in the posterior fossa due to CSF flow artefacts. However these data now warrant testing in other larger cohorts and other refinements.

The problems that affect FLAIR-derived threshold WML volume measurement are likely to affect all threshold-based techniques. Thus, in noisy MRIs or in patients with severe WMLs, a suitable area of homogeneous, normal appearing white matter may be difficult to find and a single sequence to determine the threshold is very vulnerable to RF inhomogeneities [29]. Focal lesions that create marked focal variations in signal strength, such as infarcts, large volume ventricles and haemosiderin deposits in the basal ganglia, also affected FLAIR-derived thresholds and increase WML volume variability. The MCMxxxVI method reduced considerably the influence of RF inhomogeneities and noise, improving the signal to noise ratio, thus enhancing tissue differentiation. Genuine changes in normal-appearing white matter occur with increasing age, and possibly also with increasing WMLs independently of age, and are likely to affect all threshold-based WML volume measurement and tissue segmentation techniques. Thus in subjects with more WMLs, higher thresholds may lead to an underestimation of WML lesion volume and greater misclassification of WMLs, introducing systematic bias to studies of factors associated with WML volume.
We have demonstrated that the MCMxxxVI method is fast, accurate, avoids the problems caused by many of the biological features that affect semi-automated thresholdbased segmentation of FLAIR images, and uses calculations that are relatively simple to apply. The use of two MR sequences reduces problems due to movement and field heterogeneity during scan acquisition, and the lengthy processing times and computational requirements of traditional methods. This work suggests that the multispectral colour fusion of two or more MR images is a powerful tool to be explored and considered further in tissue segmentation.

Acknowledgements This work was funded by "Help the Aged" (now part of AgeUK) and the UK Medical Research Council (funding for MVH) as part of the project "Disconnected Mind: LBC1936". KJF is funded by an MRC Clinician Scientist Fellowship award to Prof A MacLullich, and JMW in part by SINAPSE (Scottish Imaging Network. A Platform for Scientific Excellence, www.sinapse.ac.uk): a Scottish Funding Council Pooling Initiative. MVH and JMW are members of The University of Edinburgh Centre for Cognitive Ageing and Cognitive Epidemiology, part of the cross council Lifelong Health and Wellbeing Initiative. Funding from the BBSRC, EPSRC, ESRC and MRC is gratefully acknowledged. The imaging was performed in the SFC Brain Imaging Research Centre (www/sbirc.ac.uk), a SINAPSE Centre. The DICOM to Analyze image format conversion tools used were written by Dr. Paul A. Armitage. The manuscript was proof-read by Miss Natalie A. Royle. 
Open Access This article is distributed under the terms of the Creative Commons Attribution Noncommercial License which permits any noncommercial use, distribution, and reproduction in any medium, provided the original author(s) and source are credited.

\section{References}

1. Gootjes L, Teipel SJ, Zebuhr Y, Schwarz R, Leisinger G, Scheltens P, Moller HJ, Hampel H (2007) Regional distribution of white matter hyperintensities in vascular dementia, Alzheimer's disease and healthy aging. Dement Geriatr Cogn Disord 18:180188

2. Hugenschmidt CE, Peiffer AM, Kraft RA et al (2007) Relating imaging indices of white matter integrity and volume in healthy older adults. Cereb Cortex 18:433-442

3. Payne ME, Fetzer DL, MacFall JR et al (2002) Development of a semiautomated method for quantification of MRI gray and white matter lesions in geriatric subjects. Psychiatry Res Neuroimaging 115:63-77

4. Nebes RD (2002) Longitudinal increase in the volume of white matter hyperintensities in late-onset depression. Int $\mathbf{J}$ Geriatr Psychiatry 17:526-530

5. van Straaten ECW, Fazekas F, Rostrup E et al (2006) Impact of white matter hyperintensities scoring method on correlations with clinical data: the LADIS Study. Stroke 37:836-840

6. Wen W, Sachdev P (2004) The topography of white matter hyperintensities on brain MRI in healthy 60- to 64-year-old individuals. NeuroImage 22:144-154

7. Prins ND, van Straaten ECW, van Dijk EJ et al (2004) Measuring progression of cerebral white matter lesions on MRI: visual rating and volumetrics. Neurology 62:1533-1539

8. Miller DH, Barkhof F, Frank JA et al (2002) Measurement of atrophy in multiple sclerosis: pathological basis, methodological aspects and clinical relevance. Brain 125:1676-1695

9. Anbeek P, Vincken KL, van Bochove GS et al (2005) Probabilistic segmentation of brain tissue in MR imaging. NeuroImage 27:795-804
10. Wang Y, Adaly T, Kung SY et al (2008) Quantification and segmentation of brain tissues from MR images: a probabilistic neural network approach. IEEE Trans Image Process 7:11651181

11. Tohka J, Krestyannikov E, Dinov ID et al (2007) Genetic algorithms for finite mixture model based voxel classification in neuroimaging. IEEE Trans Med Imaging 26:696-711

12. Admiraal-Behloul F, van den Heuvel DMJ, Olofsen H et al (2005) Fully automatic segmentation of white matter hyperintensities in MR images of the elderly. NeuroImage 28:607-617

13. Sajja BR, Datta S, He R et al (2006) Unified approach for multiple sclerosis lesion segmentation on brain MRI. Ann Biomed Eng 34:142-151

14. Zhang Y, Brady M, Smith S (2001) Segmentation of brain MR images through a hidden Markov random field model and the expectationmaximization algorithm. IEEE Trans Med Imag 20:45-57

15. Deary IJ, Gow AJ, Taylor MD et al (2007) The Lothian Birth Cohort 1936: a study to examine influences on cognitive ageing from age 11 to age 70 and beyond. BMC Geriatr 7:28

16. MacLullich AM, Ferguson KJ, Deary IJ et al (2002) Intracranial capacity and brain volumes are associated with cognition in healthy elderly men. Neurology 59:169-174

17. Wardlaw JM, Keir SL, Dennis MS (2003) The impact of delays in computed tomography of the brain on the accuracy of diagnosis and subsequent management in patients with minor stroke. J Neurol Neurosurg Psychiatry 74:77-81

18. Fazekas F, Barkhof F, Wahlund LO et al (2002) CT and MRI rating of white matter lesions. Cerebrovasc Dis 13 (Suppl 2):31-36

19. Wardlaw J, Ferguson K, Graham C (2004) White matter hyperintensities and rating scales - observer reliability varies with lesion load. J Neurol 251:584-590
20. Ferguson KJ, MacLullich AMJ, Marshall I et al (2002) Magnetic resonance spectroscopy and cognitive function in healthy elderly men. Brain 125:2743-2749

21. Mayo Clinic(2008) Analyze 8.1. AnalyzeDirect, Inc. Mayo Clinic. http:// www.analyzedirect.com/Analyze/ upgrade.asp

22. Foley JD, van Dam A, Feiner SK et al (1996) Computer graphics: principles and practice in $\mathrm{C}$, 2nd ed. AddisonWesley Professional

23. Stockman A, Sharpe LT (2000) Spectral sensitivities of the middle- and long-wavelength sensitive cones derived from measurements in observers of known genotype. Vis Res 40:1711-1737

24. Wyszecki G, Stiles WS (1982) Color science: concepts and methods, quantitative data and formulae, 2nd edn. John Wiley and Sons, New York

25. The MathWorks, Inc. (2008) MATLAB reference manual. http://www. mathworks.com/access/helpdesk/help/ techdoc/matlab.shtml

26. Floyd RW, Steinberg L (1976) An adaptive algorithm for spatial grey scale. Proc Soc Inform Displ 17:75-77

27. Orchard MT, Bouman CA (1991) Color quantization of images. IEEE Trans Signal Process 39:2677-2690

28. Bland JM, Altman DG (1986) Statistical methods for assessing agreement between two methods of clinical measurement. Lancet i:307-310

29. Guillermaud R, Brady M (1997) Estimating the bias field of MR images. IEEE Trans Med Imag 16:238-251

30. Bedell BJ, Narayana PA, Wolinsky JS (1997) A dual approach for minimizing false lesion classifications on magnetic resonance images. Magn Reson Med 37:94-102 